\title{
New families of pure gravity waves in water of infinite depth
}

\author{
J.-M. Vanden-Broeck \\ Department of Mathematics, University College London, London WC1E 6BT, UK
}

\begin{abstract}
Nonlinear periodic gravity waves propagating at a constant velocity at the surface of a fluid of infinite depth are considered. The fluid is assumed to be inviscid and incompressible and the flow to be irrotational. It is known that there are both regular waves (for which all the crests are at the same height) and irregular waves (for which not all the crests are at the same height). We show numerically the existence of new branches of irregular waves which bifurcate from the branch of regular waves. Our results suggest there are an infinite number of such branches. In addition we found additional new branches of irregular waves which bifurcate from the previously calculated branches of irregular waves.
\end{abstract}

Keywords: nonlinear gravity waves, bifurcations

\section{Introduction}

Nonlinear periodic gravity water waves propagating at the surface of a fluid at a constant velocity $c$ have been studied for over 150 years and many interesting theoretical results have been obtained (see [1] for a review and references). Such waves can be studied by choosing a frame of reference moving with the wave speed $c$ and by assuming that the flow is potential and steady. For simplicity only waves in water of infinite depth are considered in this paper.

Stokes ([2], [3]) constructed a solution in the form of an expansion in powers of the amplitude of the wave and calculated the first few terms. Based on his results, he conjectured that, as the amplitude of the wave increases, the family of solutions will ultimately reach a limiting confitguration with a stagnation point at the crests of the waves with an enclosed angle of $120^{\circ}$. This 
conjecture was confirmed numerically by Michell [4]. Early theoretical work on the subject can be found in Levi-Civita [5] and Struik [6]. A rigourous proof of the existence of the limiting configuration was provided in [7].

Detailed numerical studies of the properties of gravity waves became possible in the second half of the 20th century. In particular the computations in [8]-[11] show that for very steep waves (i.e. waves close to the limiting configuration), $c$ is not a monotonic function of the steepness

$$
s=\frac{H}{\lambda} .
$$

Here $H$ is the difference of heights between a crest and a trough and $\lambda$ is the wavelength. More precisely it was found that $c$ goes through an infiinte succession of maxima and minima as the limiting configuration is approached. These waves were the only type of waves known up to 1980. We shall refer to them as the branch of regular waves.

Chen and Saffman [12] (see also Saffman [13]) discovered numerically new branches of solutions which bifurcate from the branch of regular waves. They refer to them as irregular waves of type $n$. They are characterised by $n$ crests per wavelength with $n-1$ crests at a different height than the remaining one. They calculated irregular waves of type 2 and 3 explicitly. These waves bifurcate from a train of regular waves of finite amplitude whose wavelengths is $n$ times the fundamental wavelength. They have limiting configurations for which the highest crests are stagnation points with an enclosed angle of $120^{\circ}$.

Olfe and Rottman [14] calculated directly the limiting configurations of irregular waves by generalising the method used by Michell [4] for the limiting configuration of regular waves. They obtained results for irregular waves of type 2, 3 and 4 .

Vanden-Broeck [15] extended the results in ([12], [14], [15]) to larger value of $n$ (up to $n=9$ ). He provided numerical evidence that the irregular waves approach a 'generalised solitary wave' as $n \rightarrow \infty$. Here a generalised solitary wave refers to a non-periodic wave characterised by a train of ripples in the far field.

In this paper we study further the properties of irregular waves. For simplicity we limit our attention to the case $n=2$. We provide numerical evidence that in addition to the bifurcation point discovered by Chen and Saffman [12], there are an infinite number of similar bifurcation points. Each 
bifurcation point occurs between two successive extrema (maximum or minimum) of the branch of regular wave. In addition we follow numerically the first branch of irregular wave with $n=2$ (i.e. the one computed by Chen and Saffman [12]) up to the limiting configuration with a $120^{\circ}$ angle at the crests and we show that this branch oscillates also infinitely often as the limiting configuration is approached. This suggests that there might be further bifurcation points between each pair of successive extrema. This is confirmed by our numerical calculations.

\section{Formulation}

We consider a train of two-dimensional periodic waves travelling at a constant velocity $c$ at the surface of a fluid of infinite depth. We take a frame of reference moving with the wave, so that the flow is steady. At infinite depth the flow is characterised by a uniform flow with a constant velocity $c$. We choose cartesian coordinates with the $y$-axis directed vertically upwards and $x=0$ at a crest or a trough of the wave. Gravity is acting in the negative $y$-direction.

We assume that the fluid is incompressible and inviscid and that the flow is irrotational. Therefore we can introduce a potential function $\phi(x, y)$ and

a streamfunction $\psi(x, y)$. Without loss of generality we choose $\psi=0$ on the free surface and $\phi=0$ at $x=0$. We also define the complex potential

$$
f=\phi+i \psi
$$

and the complex function

$$
\delta+i \beta=\frac{1}{u-i v}
$$

Here $u$ and $v$ are the horizontal and vertical components of the velocity.

On the free surface the pressure is constant and the dynamic boundary condition yields

$$
u^{2}+v^{2}+2 g y=B .
$$

Here $B$ is the Bernoulli constant. We choose the origin of $y$ to correspond to the level of the free surface where the velocity is equal to $c$. This forces $B=c^{2}$. 
We use $\phi$ and $\psi$ as independent variables. It then follows that $\delta+i \beta$ is an analytic function of $f$ in the lower half plane $\psi<0$ of the complex $f$-plane. (see for example Vanden-Broeck [1]). Furthermore

$$
x_{\phi}+i y_{\phi}=\delta+i \beta
$$

where the subscript $\phi$ denotes the partial derivative with respect to $\phi$.

We now introduce dimensionless variables by using the wavelength $\lambda$ as the unit length and $c$ as the unit velocity. The dynamic boundary condition (4) becomes in dimensionless variables

$$
\frac{1}{[\tilde{\delta}(\phi)]^{2}+[\tilde{\beta}(\phi)]^{2}}+y_{0}+\frac{4 \pi}{\mu} \int_{0}^{\phi} \tilde{\beta}(\varphi) d \varphi=1
$$

where $y_{0}$ is the value of $y$ at $\psi=\phi=0$ and

$$
\mu=\frac{2 \pi c^{2}}{g \lambda}
$$

is ta dimensionless squared Froude number. Here $\tilde{\delta}(\phi)$ and $\tilde{\beta}(\phi)$ denote the values of $\delta$ and $\beta$ on the free surface $\psi=0$. We have used (5) in (6) to express $y$ in terms of $\tilde{\beta}(\phi)$.

We restrict our attention to symmetric waves by assuming

$$
\tilde{\delta}(-\phi)=\tilde{\delta}(\phi) \text { and } \tilde{\beta}(-\phi)=-\tilde{\beta}(\phi) .
$$

Using Cauchy integral equation formula, it can be shown that $\tilde{\delta}(\phi)$ and $\tilde{\beta}(\phi)$ are related by the integral relation

$$
\tilde{\delta}(\phi)=1-\int_{0}^{1 / 2} \tilde{\beta}(\varphi)\{\cot [\pi(\varphi-\phi)]+\cot [\pi(\varphi+\phi)]\} d \varphi,
$$

where the integral is a Cauchy principal value (see for example [1] for details).

Subsituting (9) into (6) yields an integral equation for the unknown function $\tilde{\beta}(\phi)$. We shall solve this equation (referred below as the equation $A$ ) numerically.

We introduce the $N$ collocation points

$$
\phi_{I}=\frac{I-1}{2(N-1)} \quad I=1,2, \ldots, N
$$


and the unknowns

$$
\beta_{I}=\tilde{\beta}\left(\phi_{I}\right) \quad I=1,2, \ldots, N
$$

The assumed symmetry of the wave implies that $\beta_{1}=\beta_{N}=0$. We derive a system of $N$ equations for the $N$ unknowns $\mu, y_{0}$ and $\beta_{I}, I=2,3, \ldots, N-1$. We obtain $N-1$ equations by satisfying the equation $A$ at the mesh points

$$
\phi_{I}^{m}=\frac{\phi_{I+1}+\phi_{I}}{2} \quad I=1,2, \ldots, N-1
$$

where the integrals are approximated by the trapezoidal rule. (see [1] for details).

The last equation is obtained by fixing the amplitude of the wave, for example by imposing

$$
\epsilon=1-u_{0}^{2}
$$

where $\epsilon$ is given. Here $u_{0}$ is the value of $u$ at $\phi=\psi=0$. The system of $N$ nonlinear algebraic equations with $N$ unknowns is solved by Newton's method.

The choice (13) as a measure of the amplitude of the wave is more convenient than the steepness $s$ (see (1)) because the range $0 \leq \epsilon<1$ of the possible values of $\epsilon$ is known a priori. This is easily checked by noting that $\epsilon \rightarrow 0$ as the amplitude of the wave tends to zero since $u-i v \rightarrow 1$. Furthermore $\epsilon=1$ for the limiting configuration since there is then a stagnation point at the crest (i.e. $u_{0}=0$ ).

\section{Results}

The boundary integral equation method of Section 2 gives accurate results for waves of small or moderate amplitudes. However the curvature at the crests of the waves tends to infinity as the limiting configuration is approached and larger and larger values of $N$ are needed to compute accurate solutions for very steep waves. This is due to the fact that points move away from the crests as they get steeper. This problem can be overcome by concentrating mesh points near the crests. Following Chen and Saffman [12] we introduce the change of variables

$$
\phi(t)=t-\frac{\alpha}{2 \pi n} \sin 2 \pi n t
$$


where $\alpha$ is a given parameter. Here $n$ is the parameter characterising the irregular waves (see Section 1). The branch of regular wave can be computed with $n=1$.

Near the crest at $x=0,|\phi|<<1$ and (14) yields

$$
\phi(t) \approx(1-\alpha) t
$$

Relation (15) implies that points can be concentrated near the crests by choosing values of $\alpha$ close to 1 . Here we choose $\alpha=0.9999$ to compute very steep waves.

We rewrite the equation $A$ by using $t$ instead of $\phi$ as the independent variable. The derivatives and integrals with respect to $\phi$ can be rewritten as derivatives and integrals with respect to $t$ by using the chain rule. We introduce the $N$ collocation points

$$
t_{I}=\frac{I-1}{2(N-1)} \quad I=1,2, \ldots, N
$$

and the unknowns

$$
\beta_{I}^{*}=\tilde{\beta}\left[\phi\left(t_{I}\right)\right] \quad I=1,2, \ldots, N
$$

The problem is then solved numerically by following the procedure of Section 2 .

For simplicity we shall mainly concentrate on irregular waves with $n=2$. We start by recomputing the branch of regular waves by taking the wavelength to be twice the fundamental wavelength. Values of $\mu$ versus $\epsilon$ are shown in Figure 1 (see the curve starting at $\epsilon=0.1$ and extending to $\epsilon=1$ ). We refer to this curve as the main curve. As mentioned in the introduction this curve oscillates infinitely often as $\epsilon \rightarrow 1$ (i.e. as the limiting configuration is approached). The first maximum appears clearly in Figure 1.

At $\epsilon \approx 0.88$, a branch of irregular waves (with $n=2$ ) bifurcates from the branch of regular waves. The position of the bifurcation point is found by monitoring the sign of the determinant of the Jacobian matrix. This branch is also shown in Figure 1. Typical profiles are presented in Figures 2 and 3. Figure 2 shows the regular wave at the bifurcation point (i.e. the point of intersection of the two curves of Figure 1). As we move to the right from the bifurcation point to the branch of irregular waves, the crest at $x=0.5$ in Figure 1 rises on top of the height of the two other crests. This is illustrated 
in Figure 3 where we present a typical profile on the branch of irregular waves. We note that the branch of irregular waves in Figure 1 can be extended to the left of the bifurcation point. The resulting waves are simply those on the right of the bifurcation point but shifted of 0.5 in the $x$-direction. So profiles are like those of Figure 3 but with the highest crest at $x=0$ and $x=1$ instead of $x=0.5$.

The results so far are similar to those presented in [12]-[15]. We now present new families of waves. Their construction is based on the following two observations.

The first observation is that the existence of the bifurcation point in Figure 1 can be intuitively justified by the fact that there are pairs of waves travelling at the same speed $\mu$ near the first maximum of the branch of regular waves (one wave on each side of this first maximum). The wave of Figure 3 can be viewed as a superposition of such a pair of waves. This is of course a hand waving argument because superposition is not mathematically correct for the present nonlinear problem. However it suggests that the existence of irregular waves is related to the oscillations on the branch of regular waves.

The second observation is that the presence of oscillations on the branch of regular waves is related to the fact that the limiting configuration of the waves is characterised by a stagnation point at the crest with a $120^{\circ}$ degree angle. This was shown explicitly by Longuet-Higgins and Fox ([10] and [11) who found that the branch of regular waves oscillates infinitely often by perturbing asymptotically the limiting configuration with the $120^{\circ}$ angle.

The first observation suggests that further bifurcation points should be found between each successive maximum and minimum of the main curve of Figure 1. This is confirmed by our computations. Figure 4 shows a blow up of Figure 1. The first minimum of the main curve is now clearly visible. As expected a branch of irregular waves (with $n=2$ ) bifurcates from the branch of regular waves between the first maximum and the first minimum. This bifurcation was already mentioned in [16]. We found a further bifurcation point between the first minimum and the second maximum. It is shown on the extreme right of Figure 4. A further blow up (presented in Figure 5) shows this new branch more clearly. We expect that this structure continues indefinitely in the sense that there is a branch of irregular waves bifurcating from the branch of regular waves between each pair of successive extrema of the branch of regular waves. We have concentrated here on the case $n=2$ but the findings in [15] suggest that similar results to hold for $n=3,4, \ldots$.

All the branches of irregular waves ultimatey approach a limiting configu- 
ration with a stagnation point and an enclosed angle of $120^{\circ}$ at their highest crest. Therefore the second observation suggests that the branches of irregular waves also oscillate infinitely often as this limiting configuration is approached. If this is correct, then the first observation suggests the possible existence of new branches of irregular waves bifurcating from the branches of irrregular waves between each pair of successive extrema of the branches of irregular waves. These expectations are confirmed by our numerical computations as explained below.

In Figures 6 and 7 we present a blow up of the branch of irregular waves of Figure 1. These figures show clearly the first maximum and the first minimum of the expected infinite set of extrema on the branch of irregular waves. We seek new bifurcating branches by following the approach used to find the results of Figures 1-3. We first recompute the waves corresponding to the curve of Figure 6 by choosing the wavelength to be twice the fundamental wavelength. Figure 8 shows the resulting values of $\mu$ versus $\epsilon$ (presented only up to $\epsilon=0.997$ ). The values of $\mu$ are in Figure 8 are half of the values of $\mu$ in Figure 6 since $\lambda$ appears in the denominator of (7). As expected we found a new branch of irregular waves bifurcating from that branch. This branch is also shown in Figure 8. We should again expect similar branches of irregular waves bifurcating between every pair of successive extrema of the curves of Figures 6 and 7. Wave profiles of irregular waves for $\epsilon=0.9999$ are shown in Figures 9 and 10. The profile of Figure 9 corresponds to the point $\epsilon=0.9999$ on the curve of Figure 8 starting at $\epsilon=0.982$ (or more precisely on the continuation of that curve past $\epsilon=0.997)$. The profile of Figure 10 corresponds to the point $\epsilon=0.9999$ on the new bifurcating branch of Figure 8 . These two profiles are undistinguishable within graphical accuracy. However they are different as shown in the blow ups of Figures 11 and 12. In Figures 9 and 11 the three highest crests are exactly at the same height. In Figures 10 and 12 they are not. The profiles of Figures 9 and 10 were obtained with $N=1000$. We checked that these profiles do not change as $N$ is further increased.

\section{Conclusions}

We have revisited the classical problem of gravity waves propagating at the surface of a fluid of infinite depth. We have demonstrated numerically the existence of new branches of irregular waves which bifurcate from the main branch of regular waves. We have provided evidence that there are an 
infinite number of such branches. In addition we have shown that there are further branches of irregular waves with bifurcate from these new branches of irregular waves.

We have concentrated for simplicity to the case $n=2$ but we expect similar results to hold for $n=3,4, \ldots$ This is suggested by the results in $[15]$.

In conclusion we have uncovered a very complex set of bifurcations near the highest gravity wave. All the findings presented are consistent with the theoretical results in [17] and the references cited there. It is interesting to note that another complex structure (although different mathematically) was also found in [18] for standing waves.

Acknowledgements. This work was supported in part by EPSRC under grants EP/J019569 and EP/N018559.

\section{References}

[1] Vanden-Broeck, J.-M. Gravity-capillary free-surface flows Cambridge University Press 2010

[2] Stokes, G.G. On the theory of oscillatory waves Camb. Trans. Phil. Soc, 8:441-473, 1847

[3] Stokes, G.G. Supplement to a paper on the theory of oscillatory waves in Mathematical and Physical Papers Cambridge University Press, 1:314326,1880

[4] Michell, J.H. The highest wave in water Philos. Mag. 36: 430-437, 1883

[5] Levi-Civita , T. Détermination rigoureuse des ondes irrotationelles périodiques d' ampleur finie. Mathematishe Annalen 93: 264-314, 1925

[6] Struik, D.J. Détermination rigoureuse des ondes irrotationelles périodiques dans un canal à profondeur finie. Mathematishe Annalen 95: 595-634, 1926

[7] Amick, C.J., Fraenkel, L.E. and J.F. Toland. On the Stokes conjecture and the wave of extreme form. Acta Math., 2:193-214, 1982

[8] Longuet-Higgins, M.S. Integral properties of periodic gravity waves of finite amplitude. Proc. Roy. Soc. Ser. A 342: 157-174, 1975 
[9] Schwartz, L.W. and Vanden-Broeck, J.-M. Numerical solution of the exact equations for capillary-gravity waves. J. Fluid Mech. 95:119-139, 1979

[10] Longuet-Higgins, M.S. and Fox, M.J.H. Theory of the almost highestwave, The inner expansion J. Fluid Mech. 80:721-741, 1977

[11] Longuet-Higgins, M.S. and Fox, M.J.H. Theory of the almost highestwave, Part 2, Matching and analytical extension J. Fluid Mech. 85:769786, 1978

[12] Chen, B. and Saffman, P.G. Numerical evidence for the existence of new types of gravity waves on deep water. Stud. Appl. Math. 62:1-21, 1980

[13] Saffman, P.G. Long wavelength bifurcation of gravity waves on deep water J. Fluid Mech. 101: 567-581, 1980

[14] Olfe, D.B. and Rottman, J.W. Some new highest-wave solutions for deep-water waves of permanent form. J. Fluid Mech. 100: 801-810, 1980

[15] Vanden-Broeck, J.-M. On periodic and solitary pure gravity waves in water of infinite depth J. Eng. Math. 84:173-180, 2013

[16] Vanden-Broeck, J.-M. Some new gravity waves in water of finite depth. Phys. Fluids 26: 2385-2387, 1983

[17] Buffoni, B. Dancer, E.N. and Toland, J.F. The sub-harmonic bifurcation od Stokes Waves Arch. Rational Mech. Anal., 152:241-271, 2000

[18] Wilkening, J. Self-similarity at the crests of large-amplitude standing water waves. Phys. Rev. Lett. 107: 184501, 2011 


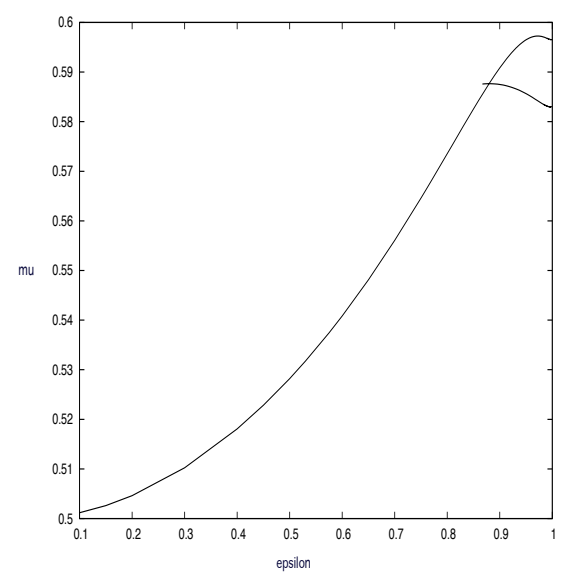

Figure 1: Values of $\mu$ versus $\epsilon$. The curve with a maximum (which is presented for values of $\epsilon$ between 0.1 and 1) corresponds to the branch of regular waves. The wavelength is here defined as twice the fundamental wavelength. The second curve at the top of the picture is a branch of irregular waves with $n=2$. This branch bifurcates from the branch of irregular wave at the point where the two branches intersect. 


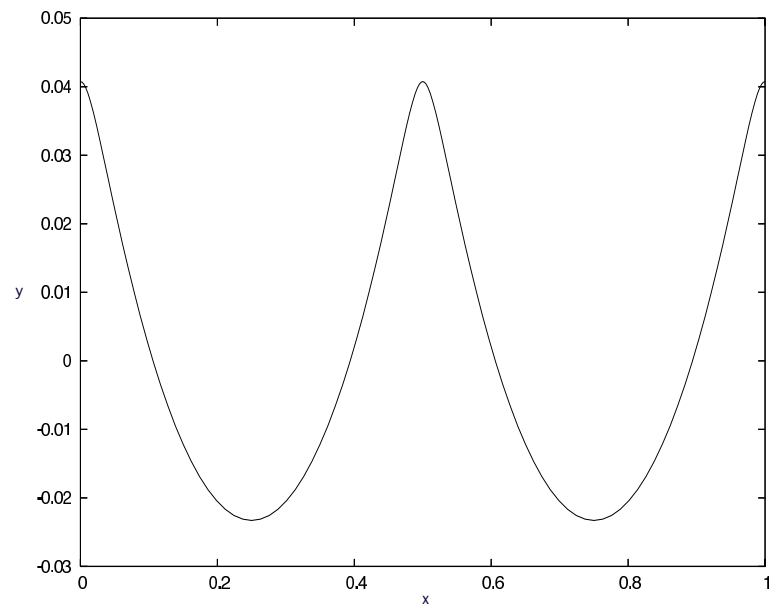

Figure 2: Profile of a regular wave corresponding to the bifurcation point in Figure 1 (i.e. the point of intersection of the two curves). Only one wavelength of the wave is shown. Here the wavelength is twice the fundamental wavelength. 


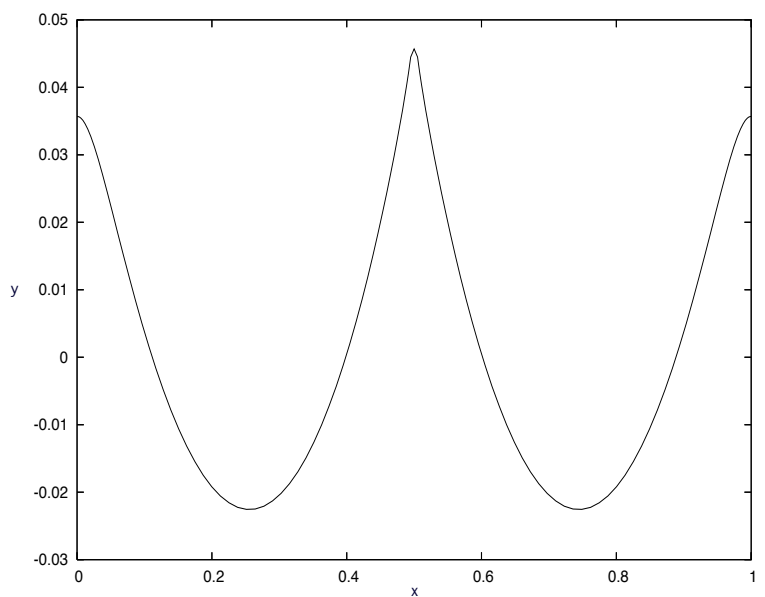

Figure 3: Typical profile of a wave on the branch of irregular waves of Figure 1. Only one wavelength of the wave is shown. 


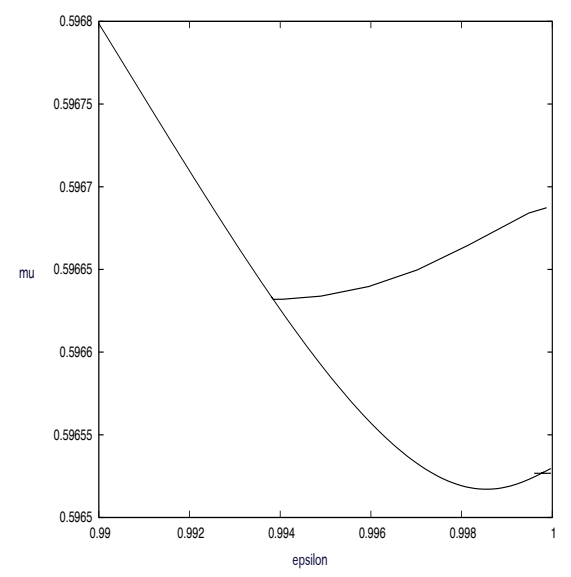

Figure 4: Blow up of Figure 1. The first minimum of the branch of regular waves is now clearly visible. A second branch of irregular waves with $n=2$, which bifurcates from the branch of regular waves just after the first maximum, is shown. A third branch of irregular waves with $n=2$, which bifurcates from the branch of regular waves just after the first minimum is also shown (see Figure 5 for further details).

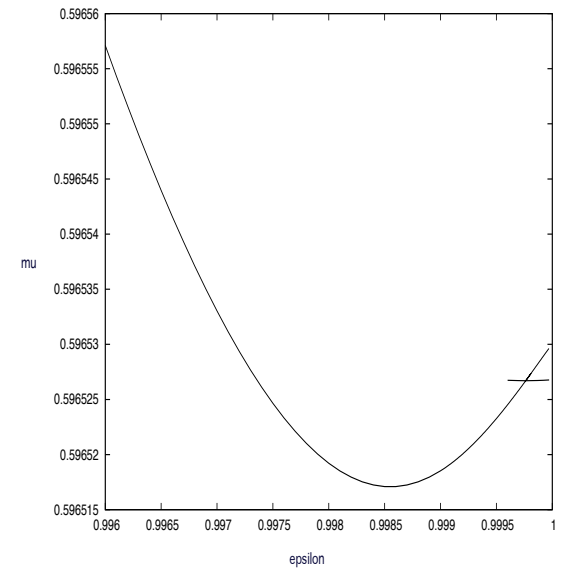

Figure 5: A further blow up of Figure 4 which shows clearly the third branch of irregular waves with $n=2$. This branch bifurcates from the branch of regular waves just after the first minimum. 


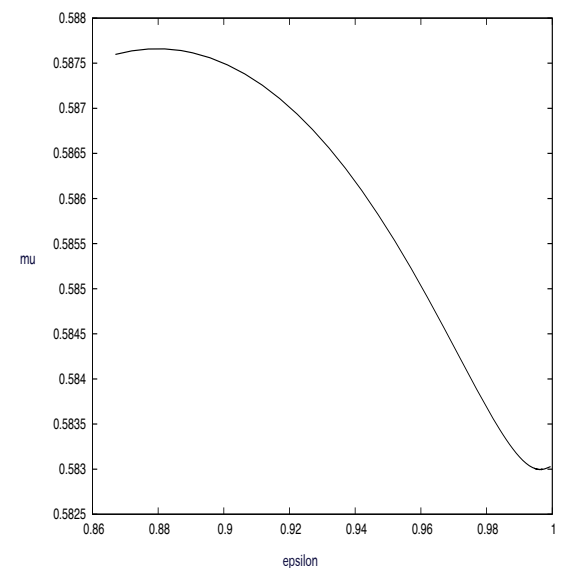

Figure 6: Blow up of the branch of irregular waves of Figure 1. The figure shows the first maximum and the first minimum. 


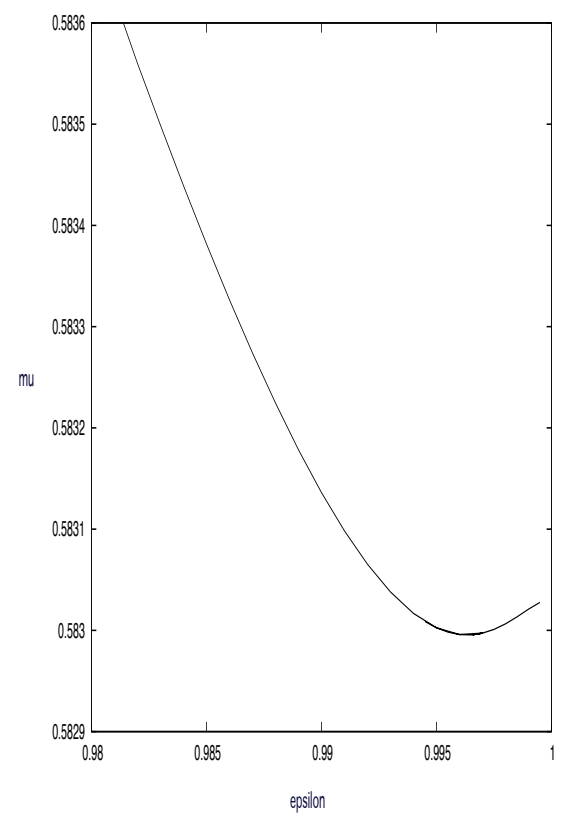

Figure 7: Blow up of Figure 6 showing more clearly the first minimum. 


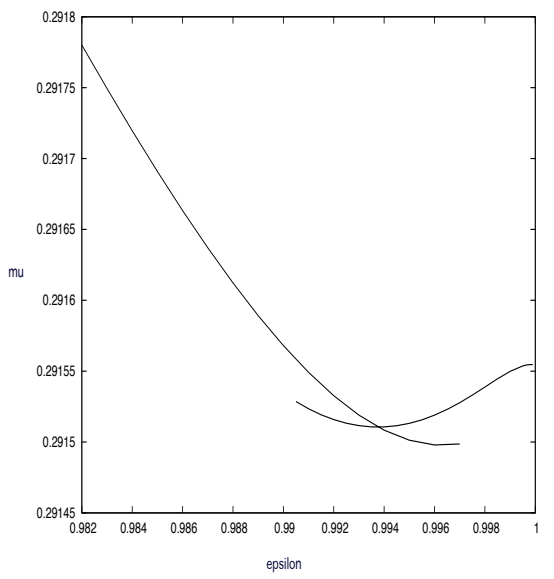

Figure 8: Values of $\mu$ versus $\epsilon$. The curve starting at $\epsilon=0.982$ is the branch of irregular waves of Figure 6. It is shown only up to $\epsilon=0.997$. The values of $\mu$ are half of those in Figure 6 because the wavelength was chosen as twice the fundamental wavelength. The other curve is a new branch of irregular waves bifurcating from the branch starting at $\epsilon=0.982$.

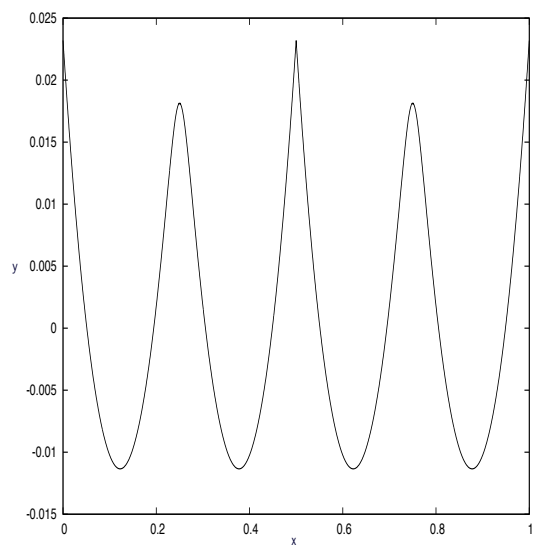

Figure 9: Profile of the irregular wave corresponding to the point $\epsilon=0.9999$ on the curve of Figure 8 starting at $\epsilon=0.982$. Only one wavelength of the wave is shown. Here the wavelength is twice the fundamental wavelength. 


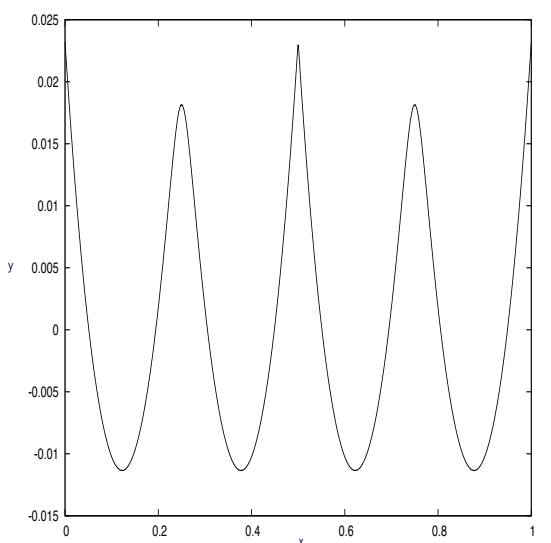

Figure 10: Profile of the irregular wave corresponding to the point $\epsilon=0.9999$ on the curve of Figure 8 terminating at $\epsilon=1$. Only one wavelength of the wave is shown.

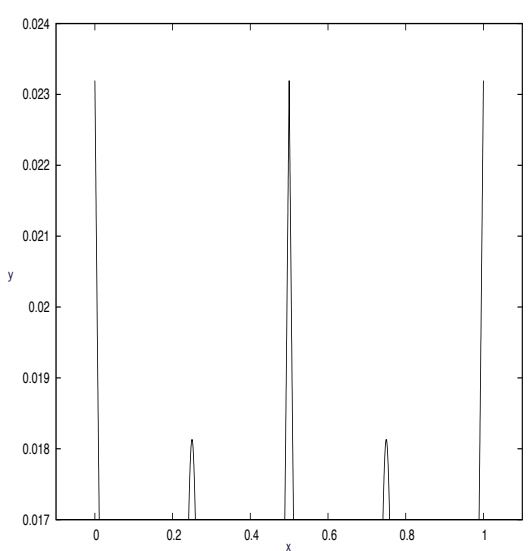

Figure 11: Bow up of the profile of Figure 9. 


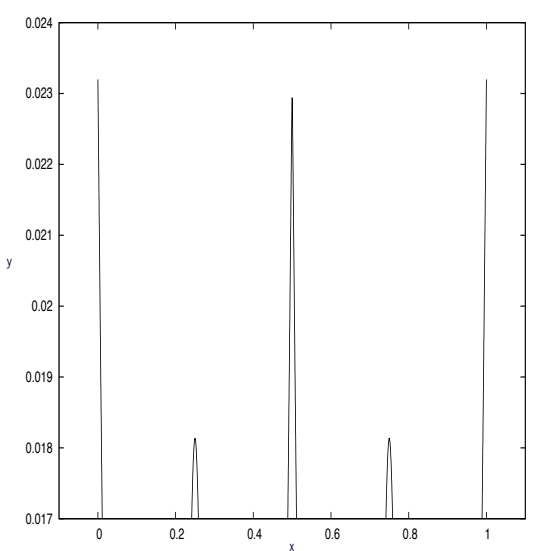

Figure 12: Blow up of the profile of Figure 10. 